


\section{REVISTA DE PSICOLOGÍA}

Vol. XXIII. Segundo semestre 2005 Nº 2

\section{CONTENIDO}

\section{ARTÍCULOS}

Denise Benatuil, Alejandro Castro Solano y Alejandro Torres. Inteligencia práctica: un instrumento para su evaluación.

Dora Herrera Paredes y Hugo Morales Córdova. Comportamiento antisocial durante la adolescencia: teoría, investigación y programas de prevención.

James S. Fleming y César Merino Soto. Medidas de simplicidad y de ajuste factorial: un enfoque para la evaluación de escalas construidas factorialmente.

Cecilia Chau y Stephan Van den Broucke. Consumo de alcohol y sus determinantes en estudiantes universitarios limeños: estudio de focus group.

Inés Kudó, Tesania Velásquez, Mónica Iza, Alicia Ángeles, César Pezo del Pino y Patricia Martínez U. Una experiencia de intervención en crisis en el sur del Perú: Brigadas Psicológicas. 
Revista de Psicología de la PUCP. Vol. XXIII, 2, 2005

\title{
Una experiencia de intervención en crisis en el sur del Perú: Brigadas Psicológicas 1
}

\author{
Inés Kudó T., Tesania Velásquez C., Mónica Iza R., Alicia Ángeles R., \\ César Pezo del Pino, Patricia Martínez U. ${ }^{2}$ \\ Pontificia Universidad Católica del Perú
}

El presente trabajo resume la experiencia de las Brigadas Psicológicas llevadas a cabo en el año 2001 como respuesta al terremoto que azotó al sur del país, causando innumerables pérdidas humanas y materiales y más de doscientos mil damnificados. ${ }^{3}$ Las brigadas congregaron alrededor de cien voluntarios entre docentes, alumnos y egresados del Departamento de Psicología de la Pontificia Universidad Católica del Perú, quienes se movilizaron a las zonas afectadas con el fin de brindar apoyo psicológico. Se presenta la intervención en conjunto: el diseño previo a la intervención propiamente dicha, las técnicas utilizadas durante la intervención y la evaluación de los resultados. Éstos incluyen el impacto en la población atendida, los factores a los que se atribuye el éxito de la intervención y el impacto en los brigadistas. La población atendida remarca de manera especial lo valioso que constituye el sentirse escuchada y acompañada, el contar con espacios para hablar y reflexionar sobre su experiencia, temores y fantasías.

Palabras clave: intervención en crisis, desastres naturales, Perú.

An experience of crisis intervention in southern Peru: Psychological Brigades

This paper summarizes the experience of the Psychological Brigades conducted in 2001 in response to an earthquake that battered the southern part of the country, causing countless human and material losses and leaving over 200 thousand victims. The brigades gathered about 100 people among faculty members, students and graduated students from the Department of Psychology of the Pontificia Universidad Católica del Perú, who went to the affected areas to provide psychological support. The complete intervention is presented, including the design previous to the intervention, techniques used during the intervention and the evaluation of the results. These include the impact on the assisted population, the factors that contributed to the intervention success, as well as the impact on the brigades' members. The assisted population specially remarks the value of feeling heard and accompanied, and of having a space to talk and meditate about its experience, fears and fantasies.

Key words: Crisis intervention, natural disasters, Peru.

1 Intervención llevada a cabo con el apoyo de la PUCP.

2 Docentes del Departamento de Psicología de la PUCP.

3 INDECI, Instituto de Defensa Civil. www.indeci.gob.pe. Las cifras exactas son 216,620 damnificados, 80 muertos y 66 desaparecidos. 

Una de las tareas fundamentales de la Psicología Clínica es promover el bienestar de las personas, lo cual supone potencializar sus capacidades y reducir aquellas interferencias que les ocasionan sufrimiento. En este sentido, resulta muy importante que los psicólogos seamos capaces de responder a las necesidades de la población y que estemos listos para actuar cuando se presentan situaciones de emergencia que requieren de nuestra intervención.

El 23 de junio del 2001, el suroeste del Perú fue sacudido por un terremoto devastador, seguido de un tsunami, afectando extensas áreas pobladas de la costa y sierra de esta parte del país. Las constantes réplicas mantuvieron a la población en un estado de alerta por varias semanas y, en la costa, con el temor de que se repita el tsunami. Las apremiantes necesidades básicas de los pobladores movilizaron la inmediata ayuda del Estado y de organizaciones no gubernamentales, nacionales e internacionales. También se hizo patente que existía demanda de asistencia psicológica por parte de las personas que vivían momentos de desolación, tensión y angustia, luego de grandes pérdidas materiales y humanas.

El Departamento de Psicología de la Pontificia Universidad Católica del Perú organizó el proyecto Brigadas Psicológicas intentando responder a esta demanda. Se trató así de canalizar los recursos de la institución y ofrecer apoyo psicológico a los damnificados. La iniciativa logró convocar rápidamente alrededor de cien personas entre docentes, estudiantes y egresados. Se tomaron en cuenta las experiencias previas de intervención en situación de crisis en las que habíamos participado: el trabajo con familiares de los rehenes de la Embajada de Japón y las Brigadas Universitarias que se llevaron a cabo en el noroeste del país a consecuencia 
de las inundaciones producidas por el Fenómeno de E1 Niño. Asimismo, se tomaron los aportes conceptuales de diferentes autores (Bellak \& Small, 1986; Bleger, 1966; Braier, 1980; Butcher et al., 1992; Cohen, 1980; Fiorini, 1975; Knobel, 1987; Pinkus, 1984), con el fin de delimitar los objetivos y técnicas a utilizarse.

A partir de esto se definieron dos principios fundamentales que sirvieron de marco a la intervención. En primer lugar, la conciencia de que el apoyo que se pudiese brindar sería temporal y por lo tanto limitado. En este sentido, la intervención sólo sería beneficiosa si se integraba a la labor que realizaban cotidianamente los promotores locales (maestros, trabajadores de salud, autoridades, líderes) y si respondía a las necesidades que ellos consideraban prioritarias, ayudándoles así en la difícil tarea de contener y dar sentido a tantas emociones desbordadas, ajenas y propias. En segundo lugar, la confianza en que los damnificados eran potencialmente capaces de resolver sus problemas, una vez que lograran identificar los obstáculos para ello. Por tanto, el apoyo ofrecido debía respetar y reforzar los estilos de cada persona o comunidad para hacer frente a las dificultades, sin imponer ni importar estrategias. La ayuda debía ser puntual y breve, a fin de desalentar la dependencia.

Sobre estas bases se diseñó el proyecto de Brigadas Psicológicas asumiendo que una intervención temprana, que brinde soporte y movilice los recursos de la misma población, constituiría una labor preventiva que redujera el impacto psicológico perturbador de la experiencia. Cuatro años después presentamos en este artículo un resumen de la experiencia completa de las Brigadas Psicológicas, su diseño e implementación y los resultados obtenidos, con el propósito de que puedan servir de referencia para futuras intervenciones ${ }^{4}$.

$4 \quad$ Se han publicado previamente algunas de las experiencias de los brigadistas: Díaz \& Navarro (2002); Kudó (2001). 
Una experiencia de intervención en crisis en el sur del Perú: Brigadas Psicológicas

Diseño: objetivos y estrategia

El proyecto se diseñó en función de dos objetivos: 1) facilitar la elaboración de la experiencia traumática a través de la contención y la escucha, y 2) activar y reforzar los recursos personales, tanto de los individuos como de la comunidad, para manejar la situación de crisis.

El marco general de la intervención contemplaba la asistencia a niños (divididos por grupos de edad), a adultos y a promotores o líderes locales. Sin embargo, durante la misma intervención, se hizo evidente la necesidad de brindar un espacio diferenciado para los adolescentes. En la medida en que se buscaba reforzar las estrategias y redes de la propia comunidad se propuso priorizar las intervenciones grupales, en la forma de grupos de apoyo breves según las urgencias y dinámicas grupales con sesiones estructuradas. Dada la demanda de los promotores de salud, se ofreció consejería individual en los casos que así lo requirieron.

La asistencia a la población siguió dos modalidades: asistencia ambulatoria (en postas, carpas u otros locales a los que voluntariamente se acercaban los pobladores) y asistencia comunitaria (los brigadistas recorrieron las localidades realizando las intervenciones en las localidades de origen o en los refugios, acompañando a las personas en sus actividades diarias). Esta última modalidad implicó un trabajo con las instituciones y autoridades locales (charlas, capacitaciones, participación en mesas de trabajo, etc.) y en algunas comunidades supuso de intervenciones comunitarias para resolver conflictos de organización de la propia comunidad o bien entre los líderes y las autoridades de la zona. 
Es importante recalcar que las estrategias planteadas en el diseño eran sólo guías generales que cada brigada debería saber adaptar en función de la configuración cultural de cada comunidad, sus necesidades y particularidades, así como también de la expertise y conocimientos de los propios brigadistas. La creatividad y disponibilidad de las y los brigadistas para adaptarse a las necesidades de los pobladores dio lugar al uso de una gran variedad de estrategias, pero siempre en el marco de la consigna fundamental de toda intervención y que era el constituirse en todo momento como espacio de escucha, contención, diálogo y reflexión. Las técnicas utilizadas fueron así sumamente variadas. En algunos casos se usaron técnicas de desensibilización sistemática para acercar a pobladores a las zonas que habían sido devastadas por el tsunami; en otros casos, particularmente con niños, se trabajó con material lúdico como títeres o caja de juego; y en otros, se usó material gráfico para la expresión de los temores o fantasías de los asistidos.

El plan de acción, basado en la experiencia de las Brigadas Universitarias ante el Fenómeno de El Niño $\left(1998^{5}\right)$, se propuso como una guía flexible para el trabajo operativo de las Brigadas Psicológicas. En el Cuadro 1 se detallan las acciones llevadas a cabo antes, durante y después de las intervenciones de campo. Como ya se señaló, cada brigada tuvo la libertad de adaptar el plan a las condiciones particulares de cada zona y a los recursos disponibles.

5 Organizadas por la Dirección Académica de Proyección Social y Extensión Universitaria de la PUCP (DAPSEU). 
Una experiencia de intervención en crisis en el sur del Perú: Brigadas Psicológicas

Cuadro 1

Plan de acción de las Brigadas Psicológicas

\begin{tabular}{|c|c|}
\hline $\begin{array}{c}\text { Intervenciones } \\
\text { de campo }\end{array}$ & Acciones \\
\hline Antes & $\begin{array}{l}\text { - Disponer los recursos necesarios para la intervención (Res- } \\
\text { ponsable de Recursos). } \\
\text { - Establecer los contactos con la zona (Coordinación General). } \\
\text { - Convocar al Responsable de Brigada (Coordinación Gene- } \\
\text { ral). } \\
\text { - Convocar a la Brigada (Responsable de Brigada). } \\
\text { - Controlar la salida de las personas, materiales y equipos } \\
\text { (Responsable de Recursos). } \\
\text { - Asamblea con todos los brigaditas voluntarios para compar- } \\
\text { tir y elaborar juntos dudas, preguntas y temores sobre la in- } \\
\text { tervención; enfatizar el uso de encuadre grupal en las inter- } \\
\text { venciones; el funcionamiento grupal de cada Brigada; y su- } \\
\text { brayar la función de escucha y sostén de las intervenciones } \\
\text { (Docentes de Psicología de la PUCP). }\end{array}$ \\
\hline Durante & $\begin{array}{l}\text { - Coordinar con las autoridades competentes en la zona (Res- } \\
\text { ponsable de Brigada). } \\
\text { - Evaluar la situación en que se encuentra la zona antes de } \\
\text { proceder (La Brigada). } \\
\text { - Realizar las actividades necesarias (La Brigada). } \\
\text { - Durante la estadía en la zona, usar permanentemente chale- } \\
\text { cos y gorras que los identifiquen como voluntarios y de la } \\
\text { Pontificia Universidad Católica del Perú. } \\
\text { - Realizar cada noche una reunión de Brigada para informar } \\
\text { sobre el trabajo por áreas, evaluar y compartir las impresio- } \\
\text { nes (La Brigada). } \\
\text { - Mantener contacto con Coordinación en Lima para decidir el } \\
\text { envío de materiales o de otra brigada (Responsable de Brigada). }\end{array}$ \\
\hline Después & $\begin{array}{l}\text { - Evaluar la experiencia (La Brigada). } \\
\text { - Presentar el informe de campo a la Coordinación General } \\
\text { (Responsable de Brigada). } \\
\text { - Supervisar la experiencia (Docentes de Psicología de la } \\
\text { PUCP). } \\
\text { - Evaluar el impacto (Coordinación General). }\end{array}$ \\
\hline
\end{tabular}


Inés Kudó, Tesania Velásquez, Mónica Iza, Alicia Ángeles, César Pezo, Patricia Martínez

Implementación

De acuerdo al plan de acción, el trabajo se realizó en tres etapas: preparación, intervención y evaluación. Durante la etapa de preparación se elaboraron diferentes propuestas de intervención, se diseñaron cartillas de apoyo y se capacitó a los brigadistas. Tomando como punto de partida la evaluación de daños realizada por los especialistas de UNICEF, se seleccionaron 58 localidades correspondientes a ocho zonas de los departamentos de Arequipa y Moquegua. Un equipo de exploración o punta de lanza visitó estas localidades con el objetivo de establecer contactos con los gobiernos locales y centros de salud, recoger las demandas de la población, verificar el acceso así como las condiciones de seguridad y alojamiento para los brigadistas. Esta etapa fue fundamental para el éxito de la intervención: fue posible contrastar la información obtenida desde Lima con las necesidades particulares de cada zona, preparar a la población para nuestra llegada y ofrecer a las brigadas información más detallada sobre el trabajo que irían a realizar así como contactos en la zona específica, a fin de articular la intervención con los esfuerzos que autoridades y otras instituciones venían realizando.

La etapa de intervención duró cuatro semanas y contó con la participación de diez brigadas. Cada brigada fue enviada a una localidad previamente designada. Las intervenciones fueron planificadas para que se lleven a cabo durante el lapso de una semana. En algunas localidades una segunda brigada fue enviada para continuar el trabajo de la anterior, según fuera la situación de crisis o una mayor densidad poblacional. Cada brigada fue conformada por entre siete y diez miembros, a cargo de un responsable general y un segundo responsable, cuidándose un balance adecuado en la composición de docentes, egresados y alumnos de los últimos años de formación en psicología. El total de miembros de nuestra comunidad educativa que participaron como voluntarios en las 
Brigadas Psicológicas fue de 88 brigadistas (16 docentes, 25 egresados y 47 estudiantes), sin contar con las personas que formaron los equipos de apoyo desde la universidad y la coordinación general.

Al llegar a la zona, cada brigada estableció los contactos con el personal de salud de la zona, las autoridades locales y otros líderes comunitarios. La intervención comenzó con un reconocimiento y una evaluación de las necesidades psicológicas de los pobladores. Los informes de las brigadas indicaron la presencia de problemáticas comunes en todos los sitios atendidos pero también dinámicas particulares en cada lugar en la manera de procesar dichas problemáticas.

La estrategia inicial estaba diseñada para trabajar con niños de manera grupal, utilizando el dibujo libre para identificar los casos que requerían una intervención. En el caso de niños entre 2 y 5 años se trabajó además con caja de juegos y títeres. La primera sesión de grupo era de carácter exploratorio y permitió establecer el encuadre. A partir de este trabajo inicial se diseñaron las actividades siguientes. Por ejemplo, en el caso de la Brigada 2, que trabajó en La Punta (poblado de escasos recursos económicos colindante al mar, arrasado por el tsunami), la intervención tuvo un fuerte énfasis en el manejo del miedo al mar. Con los niños, los brigadistas organizaron paseos y juegos, hasta que los niños estuvieron dispuestos a llegar a la orilla y ver el mar de cerca por primera vez luego del tsunami.

En el caso de los adultos se utilizaron dos posibles entradas, ambas fundadas en un encuadre grupal. La primera se usaba cuando los adultos acudían en calidad de padres de familia de niños que habían quedado "nerviosos" tras el sismo. En estos casos se partía de conversar con los padres sobre las conductas y temores de sus hijos, para luego pasar a hablar de sus propias dificultades 
y temores. La segunda se usaba cuando los adultos habían sido convocados para hablar de sus sentimientos y vivencias actuales. En estos casos se comenzaba señalando el encuadre, que incluía la presentación de los participantes y coordinadores; luego se procedía conjuntamente a una exploración de las demandas y expectativas de los asistentes; se hacía una síntesis y agrupación de los temas más recurrentes y se invitaba a compartir de forma voluntaria las experiencias vividas. Al finalizar estos relatos se hacía una revisión de los temas tratados, a modo de devolución, proporcionando información cuando era necesario, invitando siempre a la reflexión, señalando algunos aspectos, pero sobre todo reforzando la capacidad de los vecinos para acompañarse unos a otros.

La intervención de las brigadas no se limitó al ámbito clínico comunitario individual o grupal. En el proceso mismo de estas intervenciones surgieron demandas de índole social que llevaron a brigadistas a funcionar como un asesor externo a la comunidad (Bleger, 1966). Así, en algunas zonas de refugio se tuvo que apoyar en temas de organización comunitaria y resolución de conflictos de interés, para ayudar a los pobladores a manejar problemas de inclusión/exclusión entre ellos, generados por la llegada desorganizada de algunas donaciones y agentes externos.

Luego del regreso de todas las brigadas a Lima, se envió al mismo equipo de exploración para que realice una evaluación, recorriendo todas las zonas atendidas. El equipo realizó un total de 45 entrevistas (36 individuales y 9 grupales), distribuidas entre personal de salud, directores de centros educativos, autoridades, líderes locales y pobladores de todas las edades. Esta evaluación fue completada con los reportes escritos de cada brigada. Si bien no se trataba de una evaluación exhaustiva del impacto, la información recabada mostró consistencia entre los resultados reportados por las diferentes zonas y grupos a quienes se brindó asistencia. 
Una experiencia de intervención en crisis en el sur del Perú: Brigadas Psicológicas

Resultados

En términos generales la intervención tuvo un impacto positivo tanto en la población atendida como en los brigadistas. Esta sección presenta en detalle los resultados obtenidos a partir de informes de campo y de la evaluación final. Comienza con una descripción del impacto de la intervención en la población, a lo que sigue un análisis de los factores a los que atribuimos el éxito de la intervención, para concluir con el impacto en los brigadistas.

El impacto en la población

En las entrevistas post intervención se pudo identificar claramente una serie de áreas de impacto. La primera y más evidente fue el alivio sintomático en la población atendida, tanto en niños como en adultos. El personal de los centros de salud y postas médicas reportó un descenso en el número de pacientes atendidos por problemas de salud asociados a reacciones psicológicas.

Se ve a la gente más tranquila. Mi señora no dormía y ahora está más tranquila. Les ha hecho bien el conversarlo y no guardárselo (Juez de Paz, Tipan) ${ }^{6}$.

Antes en la posta lo emocional era el segundo problema en patología, venían con ataques pero era psicológico. Con decirte que se me acabó el diasepan, pero luego de que han venido ustedes ya no hay ese problema. Ahora tengo en stock (Enfermera de puesto de salud, Tagre).

6 En esta viñeta y en las sucesivas, se indica entre paréntesis el rol social de quien lo dice y, luego del coma, la localidad a la que pertenece. 
Líderes locales mencionaron el apoyo de la intervención en la resolución de algunos conflictos al interior de la comunidad. En estos casos los miembros de las brigadas cumplieron una función catalizadora al facilitar, como oído externo y neutral, el diálogo sobre los conflictos de interés. Fue una función mediadora que ayudó a los pobladores a manejar sus disputas de una manera más constructiva.

Antes habían problemas por las peleas en las familias y ahora un poquito mejor. Podemos ayudar y ser más organizados, y compartir con los otros. Algo hemos aprendido, porque no somos perfectos. Y si profesionales nos orientan, cómo no lo vamos a lograr. Ahora sólo queda un veinte por ciento de conflicto, pero antes era noventa por ciento. Teníamos que organizarnos de otra manera (Delegada vecinal de calle, Pampa Blanca).

Los padres, maestros y personal educativo resaltaron los resultados positivos del trabajo con los niños, en particular la disminución de los temores, y cómo esto facilitó el que los niños pudieran volver a desprenderse de sus padres y así retornar al colegio.

Hemos sacado niños muy afectados por el sismo y ahora sus temores han disminuido. Se ha logrado bastante en este tiempo, a pesar de que yo pensaba que era poco tiempo (Jefe de Centro de Salud, 28 de Julio).

Esa tensión que tenían los alumnos ya no está. Ahora están más centrados y se puede trabajar más tranquilo con las tareas del colegio. Estamos trabajando al cien por ciento (Director de Centro Educativo, Huami).

Asimismo hubo un impacto positivo en el personal encargado de las tareas de atención a los damnificados, siendo al mismo 
Una experiencia de intervención en crisis en el sur del Perú: Brigadas Psicológicas

tiempo pobladores de la zona afectada (promotores de salud, docentes y autoridades). En muchos casos, estas personas mostraban niveles de frustración, rabia, depresión y ansiedad más altos que el resto de la población. Esta situación es comprensible al tomar en cuenta varios factores adicionales estresantes: el doble rol de víctima y proveedor de cuidado (que a veces les impedía atender sus propias necesidades), la baja remuneración junto con las interminables horas extra sin sueldo adicional ni reconocimiento, los problemas administrativos preexistentes y, de manera especial, la falta de preparación para atender la alta demanda emocional de la población. Sus expectativas de apoyo se centraban en dos necesidades: la enorme carga emocional que debían manejar a raíz del terremoto y la sobrecarga de trabajo consecuente, que además de incrementar la presión personal los ubicaba en el "ojo de la tormenta" al interior de la comunidad.

A nosotros también nos ha servido mucho porque estábamos que volábamos. Hemos sufrido tanta presión del pueblo con eso de las donaciones, no sabíamos qué hacer. Y yo como hablando con la señorita me decía "no se preocupe" y me despejaba, y sin darme cuenta estaba trabajando conmigo (Teniente Gobernador ${ }^{7}$, La Curva).

Otro aporte del proyecto fue propiciar conciencia reflexiva sobre la importancia de los aspectos emocionales en la vida cotidiana de las personas, más allá de los generados por un desastre. El contacto con los psicólogos de las brigadas permitió a la población entender el tipo de apoyo que puede recibir de ellos y los beneficios que este apoyo tiene para el desarrollo personal.

El Teniente Gobernador es una autoridad política a nivel de circunscripción de Distrito, depende de la Prefectura Departamental y ésta a su vez del Ministerio del Interior. No es autoridad elegida, es autoridad nombrada por el gobierno de turno. 
Inés Kudó, Tesania Velásquez, Mónica Iza, Alicia Ángeles, César Pezo, Patricia Martínez

A mí lo que más me ha gustado es que traigan cómo es el trabajo del psicólogo y que los pobladores entiendan cuál es el rol del psicólogo, porque los entendían y los que han venido van a informar a otros. Ahora hay una idea muy clara de la psicología y que es un trabajo participativo (Psicóloga de Centro de Salud, San José).

A raíz de nuestra presencia en la zona, muchos médicos y enfermeras de los centros y postas de salud señalaron que se había dado un incremento en la demanda de atención psicológica por parte de la población. En todos los casos señalaron la necesidad de tener un psicólogo permanente en el centro de salud o en el colegio. De la misma manera como estos testimonios señalan con claridad los efectos positivos de la intervención en crisis, también develan la necesidad de una atención permanente y sostenida, transformada casi como una demanda social.

Factores a los que se atribuye el éxito de la intervención

Desde el punto de vista organizativo, un elemento clave para el éxito de esta experiencia fue la coordinación previa con autoridades locales y la comunicación clara de los objetivos y naturaleza de la intervención. La evaluación comprobó que los profesionales de salud y las autoridades conocían y entendían los propósitos de ésta y tenían claridad respecto a las funciones del psicólogo. Ambas condiciones permitieron que un número elevado de pobladores comprendieran el tipo de ayuda que se estaba ofreciendo. En casi todos los lugares atendidos, incluso en las zonas rurales más alejadas, los personas reconocían la importancia de una atención psicológica luego del desastre, tanto para sus hijos como para ellos mismos, y sabían con cierta exactitud los alcances de este tipo de atención. Aún en las zonas en las que había un menor conocimiento de la labor del psicólogo, el apoyo ofrecido fue 
acogido por la población luego de superar la desconfianza y desconcierto iniciales, aspecto que señala cómo, cuando se dispone de espacios de escucha psicológica, emerge la demanda latente de atención psicológica.

Todas las autoridades y promotores que fueron entrevistados manifestaron plena satisfacción respecto a las coordinaciones realizadas, pues sintieron que hubo disposición en los brigadistas para realizar nuevas tareas encomendadas. Fue crucial, en este sentido, que las brigadas estuvieran dispuestas a modificar y adaptar su plan de intervención para poder acomodarse y sostener las nuevas demandas que fueron emergiendo en el proceso de intervención. Esta actitud flexible y respetuosa permitió establecer una relación sólida con la población y sus líderes. Hay que considerar que se estaba trabajando con comunidades con altos niveles de desconfianza y expuestas a imposiciones continuas dada su vulnerabilidad: la adjudicación de un nuevo estatus (el de víctima), la asignación forzosa de una reubicación habitacional (el refugio) e, inclusive, la imposición de los términos de la ayuda recibida.

Mirando desde los pobladores, la bondad terapéutica de la intervención se puede atribuir a los siguientes factores (Kudó, 2001): 1) contar con un espacio para ser escuchados, 2) poder entender las causas del desastre y los fenómenos naturales involucrados, 3) tener la oportunidad de compartir con la comunidad, 4) la integración del humor, el juego y el afecto, 5) la permanencia, y 6) la sola presencia de profesionales que llegan de manera voluntaria.

Un aspecto que nos parece decisivo para la realización exitosa de la intervención fue la capacidad de los responsables de cada una de las brigadas para establecer, en la localidad que le tocó, una alianza de trabajo firme con los pobladores y autoridades, logrando un vínculo de confianza fundado en la credibilidad. 
Inés Kudó, Tesania Velásquez, Mónica Iza, Alicia Ángeles, César Pezo, Patricia Martínez

Este elemento merece una mención especial. Si comunidades y autoridades pudieron construir una percepción de los brigadistas como personas de fiar y profesionales capaces (con credibilidad) se debió, no sólo a la calidad humana de los brigadistas o a los contactos transparentes previos del equipo de exploración, sino al respaldo institucional que significaba estar ahí en nombre de una universidad con prestigio fundado. Este mismo argumento explica la velocidad con que la iniciativa de algunas personas fue recogida por el Departamento de Psicología, el Rectorado de la Universidad y, lo que es más importante, por un buen número de egresados, docentes y estudiantes, entusiastas, solidarios y con capacidad para comprometerse. El espíritu "católica" funcionó como elemento de amalgama y cohesión grupal que permitió fluidez en la transmisión de información entre brigadas, al interior de las brigadas y de éstas con las estructuras de apoyo y coordinación. Esta solidez grupal-institucional debe haber contribuido enormemente a que pobladores y autoridades sientan seguridad frente al interlocutor.

Un espacio para ser escuchados

La intervención de las brigadas fue reconocida como algo diferente a otras formas de ayuda.

Era la primera vez que alguien nos preguntaba por lo que habíamos vivido (Poblador, El Chorro).

Bastante ayudaba con lo que conversaban, es como sentir una paz por dentro (Poblador, refugio La Deheza-Aeropuerto).

Mi esposo recién cuando estuvieron las señoritas es por primera vez que pudo hablar y soltar todo. Ni a mí me había dicho nada. Hasta los papás se han puesto a llorar, hay personas 
que se conforman con callar y no se sabe qué están sintiendo, por eso ha sido bueno (Pobladora, refugio Punta Vieja).

El espacio de escucha y contención fue especialmente valorado por los promotores, quienes por el tipo de labores que realizaban y la sobrecarga de trabajo no tuvieron en mucho tiempo un momento para ellos mismos, un espacio para ser ellos mismos quienes recibían ayuda. Se puede confirmar el principio de las prácticas de salud mental que afirma la necesidad de espacios de elaboración afectiva de la experiencia de trabajo de la gente que trabaja con gente.

Era una forma de soltar ese momento que teníamos adentro, yo le contaba a mi compañera cómo estaba y ella a mí, pero no había nadie que nos escuchara (Enfermera de Centro de Salud, San Francisco).

En el caso de los niños ocurrió algo similar. Cuando se les preguntó qué fue lo que más les gustó del trabajo, respondieron en primer lugar "que nos preguntaran", luego mencionaron el juego y el dibujo. Es interesante notar que más allá del espacio lúdico, los niños necesitan ser escuchados y poder contar su propia versión de lo sucedido. Muchos de ellos no habían hablado del tema hasta el momento en que los psicólogos les preguntaron, principalmente porque se trataba de un tema muy sensible entre los pobladores de las zonas afectadas, sobre todo en aquellas afectadas por el tsunami. Nadie quería escuchar y por tanto hablar del miedo a la muerte, lo sorpresivo de un terremoto, lo terrorífico que resulta que el mar se salga con fuerza destructora, ni de las penas por las personas y objetos queridos y perdidos, menos de la satisfacción de haber sobrevivido.

De esta manera la intervención funcionó como un tercero cuya presencia y escucha propicia que el hablar, sintiéndose acogido, 
ayude a desanudar, desentrampar lo que está anudado, trabado, atado a la emoción traumática (por sorpresiva e intensa en su magnitud de vida o muerte). Este es un buen ejemplo de la importancia de las intervenciones psicológicas con valor terapéutico en las políticas de salud pública en casos de crisis, desastre natural o conmoción social. Pero además, al ayudar a desanudar, permite que los actores sociales que logran soltarse de estos amarres emocionales, en este caso los promotores naturales, continúen con su labor pero con una actitud nueva, menos pesada y más optimista. En ese sentido, la intervención también cumple una función pedagógica, en el sentido de la mayéutica: encontrar dentro de uno mismo los recursos para funcionar de una manera más saludable. En este contexto la manera de intervenir, la actitud terapéutica de los brigadistas, podía funcionar como un modelo al que se podía recurrir, como parte de la experiencia, para ser replicado o desarrollado por los promotores de la zona y por los mismos pobladores.

Ustedes han empezado algo y nosotros lo tenemos que continuar, como podamos. Claro, no con la misma calidad que ustedes, pero queremos resolverlo (Jefe de Centro de Salud, Moquegua).

Poder entender lo sucedido

En diferentes lugares se valoró poder comprender las causas y la lógica de los fenómenos naturales como un terremoto o un tsunami. En el caso de Camaná esto fue particularmente importante ya que el temor de un nuevo tsunami tenía a la población en permanente tensión. El valor de la información adecuada en la circunstancia propicia reafirma cómo el conocer, el saber que permite jugar en la imaginación con otros ángulos de la realidad, amplía los horizontes de la mente y asigna nuevos sentidos que ayudan a mitigar la angustia, aprender a "leer" las señales correctas 
Una experiencia de intervención en crisis en el sur del Perú: Brigadas Psicológicas

de alarma y disminuir la ansiedad paranoide que todo hecho sorpresivo e inimaginado trae.

Ante nuestros temores creíamos que de nuevo se salía el mar, y nos explicaban que primero tenía que haber un terremoto (Poblador, Santa Elizabeth Bajo).

Ahora los niños nos hablan sobre las placas, ya no están asustados. Antes ante la palabra terremoto ponían unas caritas... ahora ya se puede hablar (Enfermera de Centro de Salud, Tagre).

Debido a ciertas creencias religiosas, sumadas a ignorancias sobre los fenómenos naturales, algunos damnificados vivenciaron el desastre como un castigo divino, que rápidamente convocaba y condensaba sentimientos de culpa preexistentes y conflictos de índole moral respecto a sus vínculos con su fe y sus prácticas religiosas. Las intervenciones, al ofrecer información racional junto a una acogida afectiva, ayudaron a desculpabilizar el evento natural, permitiendo que la fuerza yóica sea puesta en las labores de emergencia y que los conflictos internos previos retomen su cause y sean tratados como tales. Por eso, informes de las brigadas reportan cómo las personas pudieron hablar de sus problemas intrapersonales o interpersonales, luego de hablar de la conmoción emocional producida por el desastre.

Hablar basado en lo científico. Porque ellos creían que eso era un castigo de Dios y eso afecta su autoestima. Era como una contradicción que vivían porque al mismo tiempo que le pedían a Dios que les proteja, sentían que todo esto pasaba porque ese Dios los estaba castigando (Enfermera de Centro de Salud, Tagre). 
Inés Kudó, Tesania Velásquez, Mónica Iza, Alicia Ángeles, César Pezo, Patricia Martínez

Una oportunidad para compartir con la comunidad

La estrategia de intervención grupal planteada en el diseño sintonizó con los problemas y recursos de la población y fue altamente valorada, puesto que implicó la posibilidad de escucharse mutuamente, de compartir problemas y analizar alternativas de solución, no sólo a nivel personal sino también comunitario. Ofrecer espacios de diálogo y reflexión grupal en intervenciones en crisis de este tipo cataliza y encausa la tendencia altruista que los desastres naturales siempre desencadenan en un primer momento (el período más cercano al epicentro emocional traumático), posibilitando que esta tendencia reactiva se transforme en una actitud consciente de solidaridad cultural, tras entender que la tragedia misma, sus efectos y la posibilidad de remediarla hasta donde es posible, es compartida. Al estar dirigida la intervención a apoyar al yo, se refuerza la función social de éste.

Lo más resaltante es que ha sido una oportunidad no personalizada sino comunitaria (Enfermera de Centro de Salud, Tagre).

También hacíamos dinámicas en grupo. La torre de papel. Y con eso pudimos captar cómo debería ser la base de cada uno y por qué se cayó, y también cómo debe ser la base de toda la comunidad (Poblador, Candelaria).

El encuadre grupal posibilitó que los damnificados redescubrieran o recrearan la experiencia comunitaria de compartir entre los miembros de la comunidad. En las diferentes zonas se expresó la idea de haber descubierto "un espacio nuevo" dentro de la comunidad. Pero en realidad "lo nuevo" estaba en la posibilidad de utilizar el espacio grupal, que es común a la condición humana, como un espacio para elaborar los afectos y pensamientos asociados a la experiencia común. En el caso de los promotores fue importante 
Una experiencia de intervención en crisis en el sur del Perú: Brigadas Psicológicas

que reconocieran la utilidad de este espacio como un instrumento profesional, al descubrir en su propio equipo de trabajo un recurso de apoyo mutuo para sí mismos y para su tarea. En ese sentido, nuestra intervención validó el funcionamiento grupal en equipos de trabajo en salud mental y en promoción del desarrollo social.

Nos hemos quedado con la idea de que tenemos que reunirnos para hablar, no sólo de cosas del trabajo, sino para compartir experiencias, eso no hacíamos antes (Jefe de Centro de Salud, Moquegua).

El humor, el juego y el afecto

En una situación de desastre que se acompaña de sentimientos de desolación, impotencia y tristeza, las herramientas del humor y el juego adquieren especial significación terapéutica para las personas afectadas. Esto es posible porque permiten procesar lo vivido de otra manera, relacionarse con los demás en un contexto y tono afectivo distintos y, entonces, compartir no sólo lo que piensan o sienten, sino también momentos de recreación que ayudan a "olvidar". Los pobladores de Camaná mencionaron las caminatas a la playa, señalando que les permitieron relajarse al mismo tiempo que le perdían miedo al mar. El efecto terapéutico sólo es posible cuando la indicación de uso de estas herramientas terapéuticas (el humor y el juego) tienen el trazo de la espontaneidad y ello sólo es posible si el vínculo asistente-asistido es genuino, no impostado. Éste es un claro signo de que los y las brigadistas supieron, probablemente de manera intuitiva, estrechar vínculos de cercanía e intimidad durante el tiempo que duró su estancia. En términos de la intervención se trataba no sólo de cumplir una función catártica y reflexiva respecto al hecho traumático teñido de muerte sino, justamente, rescatar y vivenciar los aspectos vitales y de libertad que guarda toda existencia y la 
Inés Kudó, Tesania Velásquez, Mónica Iza, Alicia Ángeles, César Pezo, Patricia Martínez

alegría de estar vivos. Muestra también cómo en estos casos, como en todos aquellos en los que la palabra no basta, el juego, la plástica y el gesto son formas de utilizar otros canales de comunicación a disposición del cuerpo para ir nombrando lo que es difícil de ser nombrado y para mantener lo que continúa vivo.

Más antes teníamos miedo, los grandes también, y ahí en la playa hemos jugado, bailado, cantado, hemos conversado (Poblador, refugio La Calderona).

Lo que nunca hemos hecho es jugar. Nuestra tensión se iba a un lado y la alegría nos lleva a otro camino, distraerse nos ayudaba (Pobladora, refugio La Calderona).

A nosotros [adultos] también nos hizo dibujar "lo que se nos venga a la mente" y todos dibujamos el desastre porque era lo que teníamos en la mente, y luego hablamos de eso. Expresábamos a través del dibujo. De una manera con las palabras a veces uno se calla, pero con el dibujo uno lo demuestra (Pobladora, refugio Punta Vieja).

En las entrevistas post intervención conmueve ver cómo los entrevistados rescataron el buen humor de "las señoritas" (las brigadistas), que los hacían reír y que propiciaban un espacio para reírse todos juntos, haciendo y provocando bromas sobre lo terrible sucedido. El humor se convirtió, en algunos casos, en un eje importante del vínculo terapéutico entre los psicólogos y los damnificados. El que las brigadas funcionaran como grupo (todas las noches se reunían para conversar lo ocurrido durante el día) permitió que elaboraran las fuertes emociones que absorbían durante el ejercicio de su función. Al no ser población damnificada podían tener una mejor distancia mental para reconocer con más facilidad la paradoja de guardar sentimientos de impotencia con la conciencia de la irreductibilidad de aspectos de la realidad. Estaban 
pues en mejor pie para transformar en chiste (vitalidad) lo inmodificable terrible (muerte, destrucción) y entonces poder trasladar espontáneamente este logro elaborado grupalmente ante la tragedia a los pobladores. Esto es importante porque señala la distinción entre un mecanismo maniaco de evasión y la transformación de lo terrible en vitalidad, cosa fundamental en situaciones de desastre que no dependen ni del control ni de la acción o inacción humana. Este destramparse de la tragedia reduce la tendencia a la victimización y abre nuevos horizontes de cómo ver las circunstancias.

Nos han ayudado bastante. Salir de la monotonía, nos reíamos, salimos de ahí y ya fue diferente. Nos hemos relajado y hasta ahora los señores se bromean con los demás (Presidente elegido de refugio, refugio Punta Nueva).

Otro elemento que fue central para los pobladores y que también tuvo un valor terapéutico fue la demostración de afecto. Los pobladores reconocieron una diferencia en el trato que recibieron de los brigadistas, frente al de los miembros de otras instituciones de ayuda. Esa diferencia radicó en muestras tangibles de cariño, en el contacto físico, en la mirada, en la comunicación gestual que patenta complicidad. Todos estos elementos son signo que la capacidad de empatía e identificación de los y las brigadistas con la población no fue perturbada por el impacto emocional de la situación de crisis que emerge en poblaciones damnificadas. Por el contrario los reportes, post intervención y de las brigadas, señalan que fueron fundamentales para la construcción del vínculo terapéutico en este contexto. Este dato confirma el precepto psicoterapéutico de que en situaciones de severa crisis aguda o crónica (situaciones traumáticas, trastornos severos por déficit, crisis psicóticas o estados psicóticos crónicos) o intervenciones psicológicas con niños, el componente de relación interpersonal genuina en la interacción asistente-asistido es, al mismo tiempo 
que inevitable, fundamental para la tarea terapéutica. Es interesante observar cómo este elemento no anula la función ni el lugar del rol del terapeuta, como lo demuestran las viñetas recogidas que al mismo tiempo que subrayan la cercanía afectiva indican la distancia de los roles ("las señoritas nos hacían reír", "han sido cariñosas", en tercera persona).

Nos han ayudado a olvidar las penas, han sido comprensivas especialmente con los niños, fue divertido, han sido muy cariñosas, es más se han hecho extrañar (Pobladora, refugio El Peaje).

\section{La permanencia}

También apareció como central el tema de la constancia, es decir, la presencia de los mismos psicólogos durante varios días, en el mismo lugar, buscando establecer vínculos sólidos con la población. Este tema tiene que ver, no sólo con la memoria respecto a las características propias de las ayudas materiales, sino con la cualidad subjetiva de un evento marcado por las pérdidas y las separaciones de personas, lugares y objetos afectivamente importantes. Los desastres naturales desorganizan un aspecto de la mente y ponen en riesgo la constancia de objeto, tan importante para la constitución o mantenimiento de la confianza básica en sí mismo y en el otro. Para el éxito de la intervención era necesario asegurar que los y las brigadistas estuvieran en lugares donde pudieran establecerse con cierta seguridad (pernoctar, alimentarse, tener su tiempo libre y su tiempo de reuniones y planificación diaria). El equipo de exploración, al establecer contactos con comunidades y autoridades, aseguró este aspecto que además de logístico tiene, como vemos, importancia terapéutica. 
Una experiencia de intervención en crisis en el sur del Perú: Brigadas Psicológicas

Una semana, todos los días han venido y estábamos con ellas todos los días. Los niños como a esta hora salían corriendo y decían "las psicólogas, las psicólogas", contentos (Líder de comunidad, Uraca).

Los días que han estado, que han sido pocos, parece que fuera un mes, dos o tres horas se quedaban hablando (Teniente Gobernadora, Chule).

Venían todos los días y eso era bueno. Cuando vinieron ellas pensamos que sólo era por un rato, un día, y por eso no dábamos interés. Pero cuando se quedaron más tiempo tomamos más interés y era bueno para nosotros (Pobladora, El Chorro).

En aquellos lugares donde la atención fue ofrecida sólo una tarde o una mañana debido a circunstancias fuera de nuestro control, en las entrevistas que realizamos post intervención encontramos dificultades para que la población recordara a los brigadistas y más aún que definiera el tipo de trabajo que realizaron o los objetivos que tenían. Mientras que en las zonas en las que nuestra intervención fue más constante, bastaba con que la población viera los "chalecos PUCP" para que los niños corrieran hacia ellos y los adultos se acercaran: "el chaleco lo dice todo, señorita".

La permanencia de las brigadas en una zona también ayudó a romper con el ciclo perverso de decepción y victimización que produce la ayuda fugaz, con frecuencia despersonalizante, que es común en situaciones de emergencia sobre todo cuando su objetivo sólo trata de cubrir la necesidad material sin ningún otro tipo de apoyo. El hecho de que los brigadistas estuvieran alojados en la zona y trabajaran diariamente con la población durante una semana o más, permitió establecer una relación estrecha con las personas, la comunidad y sus problemas, algo distinto a lo que se genera 
Inés Kudó, Tesania Velásquez, Mónica Iza, Alicia Ángeles, César Pezo, Patricia Martínez

con la sola ayuda material. Como comentó una brigadista a su regreso:

Se habían acostumbrado a ser siempre visitados por todo el mundo, pero pasaban los camiones, dejaban donaciones y no volvían más. El primer día que llegamos desconfiaban. Cuando nos vieron el segundo día, decían sorprendidos: ¡regresaron! Al tercer día, vieron permanencia... y empezaron a confiar. Nos dejaron entrar a sus carpas (Brigadista).

La permanencia permite trabajar más allá del impacto inicial del recuerdo y narración de lo sucedido. Permite la elaboración psíquica y encontrar sentidos, facilitando la superación de sentimientos de angustia y tristeza. En las intervenciones más cortas no alcanza el tiempo psicológico necesario para reorganizar la experiencia luego de ser removida por el primer acercamiento. Aquí se puede constatar cómo intervenciones psicoterapéuticas o aquellas que implican acción cultural, son procesos que reclaman un tiempo cronológico para poder desplegarse y cumplir su función. No es una cuestión de compromiso con el desvalido sino un criterio técnico del encuadre. Si un encuadre es invariante -sea el que se fije- y es respetado por el que asiste, los procesos despertados en el asistido y el proceso del vínculo con él se pueden desplegar por caminos más saludables.

Al comienzo, mientras hablamos, nos sentimos mal por recordar, como si en ese instante lo volveríamos a pasar, pero luego nos sentimos bien (Poblador, Chucarapi).

El impacto de la sola presencia

El solo hecho de que profesionales de otro lugar se tomen el tiempo y el esfuerzo para llegar hasta donde se encontraba la 
población fue, en sí mismo, reparador para los damnificados. Significaba que un otro diferente y ajeno reconociera de su existencia y de sus necesidades. Los pobladores valoraron la entrega y el compromiso desinteresado de los brigadistas, el que sin conocerlos hayan estado dispuestos a viajar "desde tan lejos" para prestarles ayuda, cuando a veces ni siquiera entre vecinos hay disposición para apoyarse mutuamente. En zonas rurales alejadas, como Tipan y Viraco que quedaron aisladas por un largo período luego del terremoto, esto fue especialmente importante pues vivieron con mayor intensidad el "abandono" por parte de autoridades y de la sociedad en general.

Lo que nos ha gustado y nos ha hecho sentir bien es que personas de afuera se interesaron por nosotros, que sin conocernos nos preguntaron cómo nos sentíamos y que les importara. La ayuda desinteresada, y que vinieran de tan lejos con su paciencia para escucharnos a cada uno... esto nos ha hecho pensar sobre cómo no nos preocupamos por las personas que están a nuestro lado (Teniente Gobernadora, Chule).

Para mí lo más importante, aunque no haya habido intervención, lo más importante es su presencia. Es darnos cuenta de que no estamos olvidados, uno siente más confianza y el valor, saber que no estamos solos. Su visita es sentir que el Perú y el mundo está con nosotros y nos viene a ver (Poblador, Turpayto).

Aquí cabe reflexionar sobre los resortes de los miembros de una sociedad para acudir en apoyo de conciudadanos que han sufrido una desgracia de magnitud. Se juega no sólo la capacidad de solidaridad de las personas individuales sino el grado de cohesión social de un país. Asuntos de carácter político y cultural que son de máxima importancia en una sociedad como la nuestra, tan diversa culturalmente y tan marcada por inequidad y severas brechas 
Inés Kudó, Tesania Velásquez, Mónica Iza, Alicia Ángeles, César Pezo, Patricia Martínez

socioeconómicas. Pero en términos específicos de salud mental qué importante resulta para personas afectadas por situaciones de desastre o conmoción social, saber que pueden contar con conciudadanos desconocidos personalmente. Esto no sólo refuerza sentimientos de pertenencia a una comunidad mayor, sino que contrarresta la fuerza destructiva de los sentimientos de abandono y desolación. Confirma que en situaciones emocionales de desvalidez, la presencia de un otro funciona como sostén y ternura que ayuda a resarcirse y reencontrarse con las propias capacidades de recuperación. Es como vislumbrar una luz al final del túnel.

Una alianza de trabajo basada en la credibilidad

En las entrevistas post intervención se recogieron múltiples comentarios sobre la calidad personal y profesional de los brigadistas. La población destacó la disposición que mostraron para apoyar en todo lo que se necesitaba y podían hacer, junto con la voluntad de trabajar cuantas horas fuesen necesarias, atender en cuantos lugares así lo requiriesen y hacer todo esto con buen humor y mucha energía.

Una característica de los y las brigadistas que fue especialmente valorada por pobladores y promotores, fue su sencillez y la capacidad de relacionarse con las personas de una manera horizontal, tratando de utilizar un lenguaje claro y genuino, sin colocarse en una posición de superioridad y sin discriminar a nadie. Nuevamente hacen mención al buen ánimo y a la calidez en el vínculo.

En esto hubo una feliz sintonía entre las características individuales y grupales de los y las voluntarias que conformaron las brigadas y las necesidades afectivas de la población apoyada y el tipo de trabajo encomendado. En situaciones de crisis un aspecto 
fundamental del encuadre clínico es la interacción real de la persona que asiste con las personas que son asistidas. En este tipo de circunstancias se pone en relieve el aforismo psicoterapéutico de que el principal instrumento de toda intervención psicológica es la persona misma de quien interviene, la síntesis personal que haya logrado de experiencia vital, conocimientos teóricos y técnica. Al mismo tiempo subraya, en contraste, que la impostación defensiva que se ocupa de generar una distancia artificial rigidiza el sentir, el pensar y el actuar adecuados a la situación.

Porque para reuniones para reestablecernos del terremoto la gente no venía, se iba de las reuniones. Pero con ellos sí se trabajó en grupo, lograban reunir a la gente. Es que lo hicieron muy atrayente y todos tenían un carisma especial, eran como uno de ellos de la población, rápidamente se familiarizaron con los psicólogos (Enfermera de Centro de Salud, Tagre).

Las señoritas se han hecho querer, creo que por el modo de comportarse y tratar por igual a la persona, sin distinciones (Esposa del Alcalde, Corire).

Tanto ha sido su carisma que la población los ha aceptado, en la posta, en los colegios, en las plataformas, hay un buen concepto (Jefe de Centro de Salud, 28 Julio).

La sencillez también tiene que ver con la posibilidad de adaptarse a todo tipo de situaciones, de trabajar en cualquier espacio, de tolerar la incomodidad del alojamiento, de comer la comida que les ofrecieran y vincularse con todos los que así lo quisieran sin discriminación alguna. Aquí hay dos elementos a subrayar (empatía y encuadre) que adquieren variaciones en intervenciones en crisis por circunstancias como desastre natural o conmoción social. En estas situaciones la empatía no es sólo ponerse en el lugar 
del otro y tratar así de ayudarlo a desplegarse, es también tener que vivir semejante circunstancia de vida mientras se esté presente en el lugar de los hechos. No es una elección, es condición inherente a la labor de un voluntario en zonas de emergencia. Otro elemento que confirma esta experiencia tiene que ver con que el aspecto mental del encuadre cobra mayor relevancia que el aspecto espacial (concreto) del mismo. En circunstancias precarias el encuadre es finalmente todo ambiente posible. Se puede precisar espacios, tratar de mantenerlos pues son convenientes para sostener intimidades, pero tener la plasticidad de moverse según los movimientos de la población afectada que se atiende resulta indispensable. La función principal (el encuadre mental) de toda intervención en crisis es cumplir una función de contención (con toda su reminiscencia del vínculo temprano) y apoyar al fortalecimiento del yo, funcionando inclusive como yo auxiliar. Teniendo claro este objetivo terapéutico, una playa, un sendero, un patio u otro lugar son espacios donde también se despliegan contención y apoyo. Esta multiplicidad temporal y espacial del encuadre en situaciones de emergencia social explica lo agotadoras que resultan las jornadas diarias y la importancia de contar con un grupo-equipo de trabajo que funciona como espacio mental de contención y elaboración del equipo asistencial. De ahí la importancia de que en cada brigada hubiera un balance entre experiencia y juventud.

A ellos no les importaba dónde era, suelo, pista, cerro. Y se ponían al nivel de los niños a trabajar con toda dedicación y era bonito.

Nos fuimos a Yacme caminando con el sol, y es pesado porque es subida. Nosotros normalmente no hacemos ese camino y yo pensaba "cómo los estamos trayendo", pero ellos felices caminaban (Enfermera de Centro de Salud, Tipan).

Cuando iban allá a Villa Hermosa les ofrecían de comer, ellas tenían su pensión acá, pero igual comían con gusto y 
decían "qué rico que está", aunque no lo fuera, porque eran de la olla común. Pero ellas aceptaban todo lo que les dieran (Esposa de Alcalde, Corire).

Mencionaron también el profesionalismo y la calidad del trabajo psicológico, señalando que tenían muy claro lo que iban a hacer. Sin embargo, si bien hay total satisfacción respecto al desempeño profesional, las características más valoradas de los brigadistas fueron las que daban cuenta de su calidad humana, su entrega, su espontaneidad y el cariño con los que asumieron su trabajo y con que se relacionaron con la población. Definitivamente, desde el momento mismo en que se enrolaron en este voluntariado especializado, las personas lo hicieron con espíritu de compromiso y aventura, abiertos a lo desconocido y a lo incierto, tan importante para cualquier experiencia de encuentro con un otro diferente, más si éste es lejano y confronta un desastre.

No solamente era la psicóloga o el psicólogo, sino que son personas. Muy puros, muy dadivosos y cariñosos. Nos hemos quedado impresionados, no sólo con el profesional, sino con la persona. Nos hemos quedado encantadísimos. Nos interesa no sólo que sean excelentes en la tarea, sino que sean buenas personas y quieran trabajar (Enfermera de Centro de Salud, Tipan).

Desde el otro lado: el impacto en los brigadistas

Si algo puede caracterizar las sensaciones de los brigadistas durante esta experiencia es la incertidumbre y la duda. Es importante señalar que en la mayoría de casos no se trataba de psicólogos experimentados en intervención en crisis, mucho menos familiarizados con el contexto devastador de un desastre natural de la magnitud de este sismo. Si cada brigadista estuvo en capacidad de enfrentar los retos de la tarea, fue porque contaba con preparación 
profesional suficiente y, sobre todo, con el soporte indispensable de su propia brigada que tenía la consigna de tratar de funcionar siempre como un grupo-equipo. Cada brigada funcionó como condensación de todos los soportes -emocional, intelectual e institucional- que necesitaba para cumplir con su tarea. Cada brigada configuró su propia dinámica pero en todas funcionaba una estructura de respaldo y contención necesarios. Constituirse como un grupo, un espacio permanente de elaboración de emociones, afectos y dudas daba el tramado psicosocial necesario para encontrar la respuesta profesional posible en cada momento específico. Saber que eran parte de un esfuerzo institucional y que contaban con ella ante cualquier circunstancia que pusiera en riesgo su integridad, fue tan importante como el establecimiento previo de contactos y sistema de comunicación que el equipo de exploración fijó. Aún así, la incertidumbre fue una parte, a la vez que natural, necesaria de la experiencia. Permitió a los brigadistas afrontar con honestidad las limitaciones de su trabajo y lo que como personas podían dar. Todas situaciones en crisis al mismo tiempo que son semejantes (sorpresa, miedo, pérdidas, temor a la repetición) son singulares como lo son las personas afectadas. Contener y apoyar al yo tiene como requisito estar siempre receptivos a lo que la población comunica, a los emergentes que se detectan y que siempre son nuevos. Las intervenciones en crisis son un constante aprendizaje, tanto de la problemática como de lo que uno puede dar y de los límites que uno tiene. Las situaciones de emergencia social muestran los límites de lo conocido y ponen a prueba cuán capaces somos de transformar lo conocido limitado en creatividad e intervención eficaz para ese aquí y ahora irrepetible.

Yo quiero comentar que definitivamente me angustia la pobreza, las necesidades y las experiencias de esta gente que intentamos ayudar, pero que en su evidente deseo de ser escuchados encuentro la pauta que dan, mostrándonos cómo podemos serles de alguna utilidad. 
Las estudiantes de básica nos decían que les movía no poder hacerlo, no poder responder como se necesita. Y las mayores decíamos "a nosotros también". Porque era esa la sensación de todas.

Las emociones experimentadas durante el proceso fueron muy intensas, como lo expresan los diarios de campo y los testimonios de algunos brigadistas. Esto es comprensible pues luego de que cede la inicial y natural desconfianza y un equipo de salud mental comunitaria es aceptado por la población devastada, el equipo se transforma rápidamente en la metáfora de una antena o imán. Si una población tiene necesidades urgentes de índole psicológica y tiene a la mano la posibilidad de un servicio, las necesidades se transforman rápidamente en demanda básicamente emocional, con todo lo que esto trae (confusión, desasosiego, pena, temor, culpa, incertidumbre, etc.). Los equipos de atención son velozmente inoculados de una masa de narraciones, balbuceos, gestos y silencios llenos de una emoción a veces informe y difícil de nombrar. Los especialistas que atienden son personas y tienen un doble trabajo mental: digerir en su propio interior las propias emociones que han sido agitadas al recibir la magnitud (cuantitativa) de emociones intensas (cualitativa) de otros que sufren por realidades irreductibles que han desorganizado el equilibrio previo que tenían. Alcanzada cierta digestión (o elaboración), el asistente, él solo o en equipo, debe tratar de encontrar la manera más sencilla y veraz de ayudar al asistido a digerir aquellas emociones que le comunicó. Contener y apoyar al yo del asistido, de manera individual y grupal, pasa por contener y apoyar al propio yo del asistente:

Es realmente difícil, realmente difícil encontrar las salidas; así creo que se siente cada señora, señor o niñito. En medio de tanta carencia, de tanta desgracia, las personas están agradecidas de que estemos cerca de ellos, eso es genial y 
Inés Kudó, Tesania Velásquez, Mónica Iza, Alicia Ángeles, César Pezo, Patricia Martínez

por eso me siento contenta aunque no pueda resolver los problemas.

Siento muchas cosas entre felicidad, impotencia y deseos de hacer muchas cosas. Creo que algo se ha comenzado y es lo que hay que continuar.

Las lágrimas en los ojos de los esposos fueron realmente muy... no sé, muy fuertes... han dejado un montón de sentimientos encontrados en mí, que sólo espero comprender en el transcurso de estos días que nos quedan con ellos.

La clave en todo este proceso fue la capacidad de manejar las emociones manifestadas en forma de dudas de una manera abierta, libre, y utilizar el equipo de la brigada como un espacio grupal para el soporte y contención intra e interindividual y para hacer del equipo el espacio de supervisión grupal de las tareas a llevar adelante antes, durante y después de las intervenciones. En este sentido, un criterio principal para elegir a responsables de brigada fue la conjunción de experiencia en el manejo de grupos y cualidad para constituirse en líder natural de la brigada. Pero un jefe de brigada no puede estar solo y fue por ello que tenía un segundo, un par que cumpliera la función de co-coordiinación, es decir, formar parte del primer núcleo de elaboración y retroalimentación que fijará el perímetro de solidez mental de todo el equipo. Los otros criterios que se utilizaron para elegir al primer y segundo responsable de cada brigada fueron: tener una mayor experiencia clínica, comunitaria o no, ser docente o egresado (es decir, ya psicólogos profesionales) y aceptación del encargo.

Para terminar, no está demás volver a subrayar que esta experiencia de las "Brigadas Psicológicas", llevada a cabo de manera colectiva, tuvo un impacto profundo individual en cada uno de los voluntarios, tanto como un mayor aprendizaje de las propias hablidades 
profesionales como en términos de un crecimiento humano, con significaciones personales muy variadas. Para las personas y para nuestra institución ha sido una experiencia de gran significado cultural, al permitir este proceso de ida y vuelta que es el aplicar y aprender en una situación real, que además nos permite mirarnos y mirar personas y lugares de nuestro país que están alejadas (mental y geográficamente) del ámbito familiar de la universidad.

Esta experiencia ha cambiado mi vida. Ahora soy otra persona.

Ha sido la experiencia humana más alucinante de mi vida. La más fuerte que he vivido.

El trabajo realizado por nuestra brigada en la provincia de Camaná, Arequipa, entre el 22 y 28 de julio del 2001 a raíz del terremoto y el tsunami que afectó esta zona ha constituido una experiencia crucial en nuestra historia profesional. Hemos podido aproximarnos al trabajo en crisis por primera vez, pero además conocer más de cerca la realidad psicológica fuera de Lima.

Reflexiones finales

Nuestra presencia en las localidades afectadas nos permitió comprobar cómo los pobladores debían lidiar con las consecuencias no de uno sino de tres terremotos. El terremoto es un evento que irrumpe en la vida de una comunidad y destruye, trae consigo angustia y desolación. Lo sigue la respuesta de la sociedad civil y del Estado, que genera una dinámica propia: la dinámica de la ayuda. Su presencia es vivida de forma disruptiva, dando lugar a reacciones y sentimientos de angustia e ira, conflictos de interés entre la población, actitudes de pasividad y dependencia. Ambas 
Inés Kudó, Tesania Velásquez, Mónica Iza, Alicia Ángeles, César Pezo, Patricia Martínez

situaciones reactivan un problema crónico y de base: el terremoto de la pobreza.

El terremoto en sí

Tanto el terremoto como el tsunami ocasionaron cuantiosas pérdidas materiales (hábitat y pertenencias con valor afectivo), a las que se sumó la pérdida de los medios de subsistencia (destrucción de medios de producción y de trabajo redituable). Pero también una pérdida del equilibrio emocional previo que llevó a un conjunto de desorganizaciones internas traducidas en síntomas o mecanismos de defensa fallidos o retardados en su función adaptativa. Los registros realizados por los brigadistas permitieron identificar la presencia de diversos síntomas en la población (niños, niñas, adolescentes y adultos), entre los que destacan: los síntomas somatomorfos, hipertrofia del estado natural de alerta y alteraciones del sueño y la alimentación. La depresión fue el trastorno más diagnosticado y los sentimientos prevalentes eran miedo, culpa, rabia y, en las madres, una preocupación intensa por los hijos. En las zonas de costa afectadas por el tsunami fue frecuente encontrar niños con temor al mar que se resistían a acercarse a la orilla o a volver a sus casas, especialmente de noche.

El terremoto de la ayuda

El despliegue de donaciones que la catástrofe movilizó de parte del Estado y otras organizaciones fue vivido en muchas zonas del sur como un elemento externo que irrumpía con violencia en la comunidad, casi como produciendo un segundo terremoto. Según pudimos observar, la ayuda se puede convertir en un problema severo cuando es desorganizada (inconstante, a veces excesiva, insuficiente o inservible) o cuando introduce violentamente 
Una experiencia de intervención en crisis en el sur del Perú: Brigadas Psicológicas

elementos ajenos a la zona, es decir, cuando no responde a las necesidades reales de la población o es brindada de manera tal que desborda la capacidad administrativa de las autoridades locales. Este nuevo terremoto lo que produce son conflictos sociales intensos (de interés, de inclusión/exclusión, de rivalidad innecesaria) entre los pobladores o bien de éstos con las autoridades (dado que se vive con suspicacia la mala distribución de los donativos) además de promover la pasividad y la dependencia. En este último caso, la ayuda substituye y por tanto erosiona la capacidad de respuesta de la propia comunidad, se favorece la posibilidad de victimización, una actitud de espera y fragilidad colectiva que ataca los resortes de cohesión y solidaridad social.

El terremoto de la pobreza

Sin embargo la confirmación más significativa tiene que ver con la manera cómo el impacto psicológico y social del desastre natural fue amplificado por las condiciones crónicas de pobreza en las que viven vastos sectores de la población peruana. El fenómeno natural pone al descubierto que la pobreza no sólo consiste en la falta de recursos y condiciones socioeconómicas básicas para vivir de una manera digna (vías de comunicación, servicios de salud, educación e infraestructura sanitaria y de seguridad ciudadana), sino que subraya las características de la violencia social y familiar, las cadenas de dominación y exclusión.

Desastres naturales, apoyo psicológico y responsabilidad social universitaria

En el Perú las estrategias de apoyo a las poblaciones damnificadas por desastres naturales rara vez contemplan la atención psicológica. El impacto visible de un terremoto o tsunami es tan intenso 
Inés Kudó, Tesania Velásquez, Mónica Iza, Alicia Ángeles, César Pezo, Patricia Martínez

que la reacción inmediata es atender las necesidades materiales, sin duda prioritarias pero no las únicas.

Sin embargo, la experiencia de las Brigadas Psicológicas nos permite constatar que existe urgencia en los damnificados de espacios para elaborar las situaciones traumáticas que han atravesado y encontrar estrategias para lidiar con las pérdidas afectivamente significativas y la precariedad del existir. Si bien estas urgencias pueden no ser reconocidas inicialmente como necesidades por los propios pobladores (abrumados por las carencias materiales, los temores y la desconfianza), rápidamente se crea una demanda cuando se brinda espacios de ayuda psicológica.

Para que esta intervención sea beneficiosa y no constituya un nuevo acto de violencia hacia la población, resultan esenciales la escucha atenta y la planificación. Escucha como capacidad para captar las necesidades reales de los pobladores y la manera como desean ser ayudados. Planificación para brindar una asistencia organizada y articulada con las redes locales, que no aliente la dependencia sino que por el contrario fortalezca los propios recursos comunitarios de la población.

Las instituciones de docencia tienen una responsabilidad social importante frente a este tipo de acontecimientos, ya que cuentan con los recursos materiales, humanos y conocimientos que se requieren para este tipo de intervenciones. Si una institución universitaria conjuga la participación de sus docentes y profesionales egresados, la importancia que adicionalmente este tipo de experiencias tiene para los psicólogos en formación es invalorable (ver en el Anexo B el testimonio del Coordinador General). 
Una experiencia de intervención en crisis en el sur del Perú: Brigadas Psicológicas

Referencias

Bellak, L. \& Small, L. (1986). Psicoterapia breve y de emergencia. México: Manual Moderno.

Bleger, J. (1966). Psicohigiene y psicología institucional. Buenos Aires: Paidós

Braier, E. (1980). Psicoterapia breve de orientación psicoanalítica . Buenos Aires: Paidós.

Butcher, J., Stelmachers, T. \& Maudal, G. (1992). Intervención en crisis y psicoterapia de urgencia. En I. Weiner, Métodos en Psicología Clínica. México: Limusa.

Cohen, R. (1990). Manual de atención de salud mental para víctimas de desastres. México: Harla.

Díaz, T. \& Navarro, R. (2002). Las Brigadas Psicológicas en Arequipa: una experiencia de intervención en crisis con niños y adolescentes. Transiciones, 4, 77-25.

Fiorini, H. (1975). Teoría y técnica de psicoterapia. Buenos Aires: Nueva Visión.

Knobel, M. (1987). Psicoterapia breve. Buenos Aires: Centro Editor de América Latina.

Kudó, I. (2001). Las huellas de un desastre. Intervención psicológica en el sur. Cuestión de Estado, 29, 85-86.

Pinkus, L. (1984). Teoría de la psicoterapia analítica breve . Buenos Aires: Gedisa. 
Inés Kudó, Tesania Velásquez, Mónica Iza, Alicia Ángeles, César Pezo, Patricia Martínez

ANEXO A

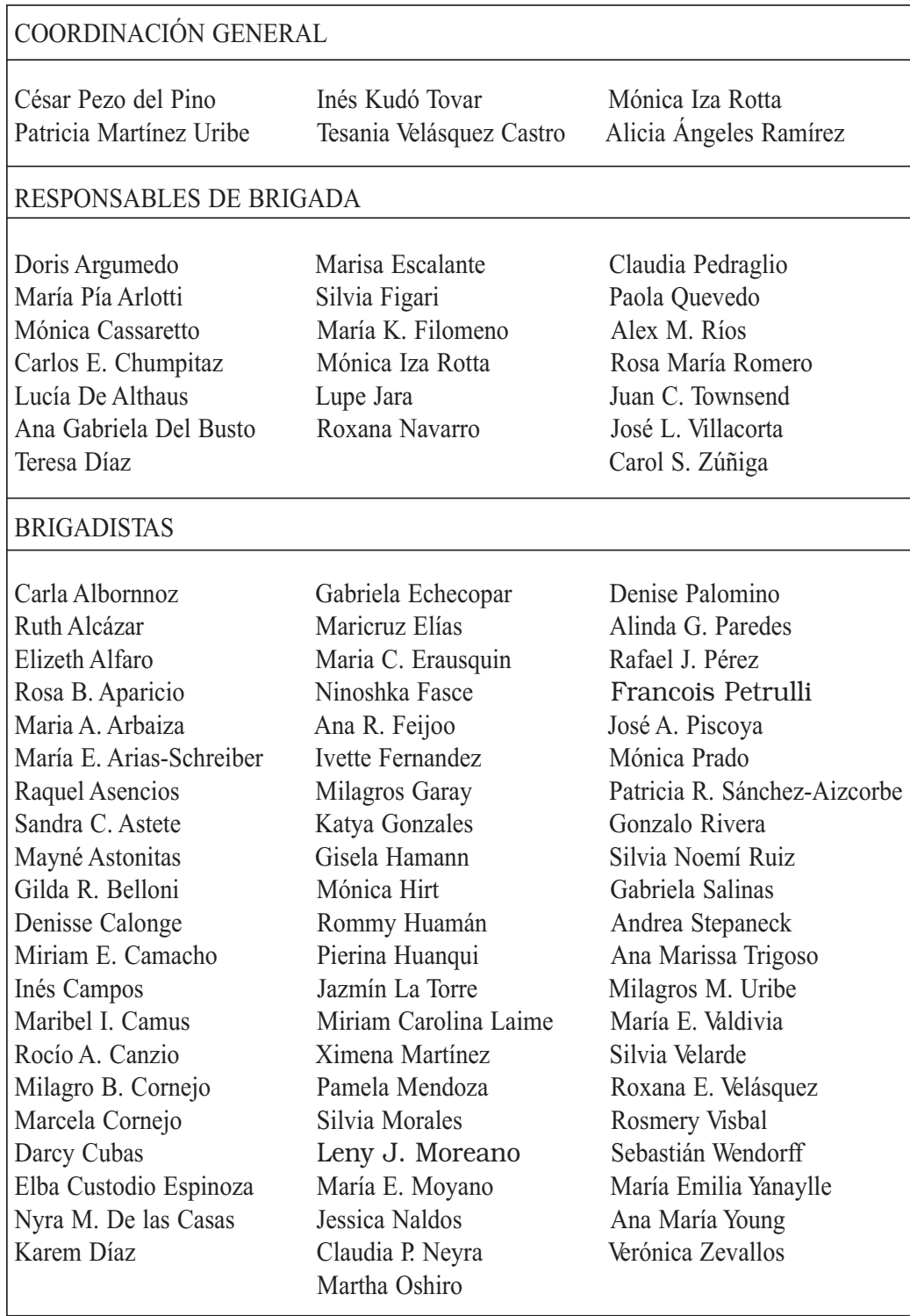


Una experiencia de intervención en crisis en el sur del Perú: Brigadas Psicológicas

EQUIPO DE APOYO

Mónica Gayoso Hugo Morales

María N. Vila

Carlos Iberico

Jorge E. Rivas

Mariana Yglesias

Pierina Traverso

\section{ANEXO B}

Significado de las Brigadas

César Pezo del Pino ${ }^{8}$

El equipo de coordinación me ha pedido que diga algunas palabras sobre el significado de esta experiencia.

Como es obvio no tengo nada que decirles sobre lo que ustedes mismos han vivido en los días previos a su salida. Menos aún sobre la gran experiencia que han tenido en el contacto directo con las personas con rostro, nombre y sufrimiento propios. Y poco sobre la intensidad de los afectos, emociones y comprensión intelectual que cada uno de ustedes guarda, que tendrán que seguir elaborando en grupo -pensando y sintiendo- y que estoy seguro se traducirá en textos que comuniquen esta valiosa e insustituible experiencia profesional y humana a otros colegas e interesados.

Sólo me voy a limitar a subrayar algunos elementos de nuestro entorno institucional y clima cultural que han hecho posible en

8 Coordinador de la Unidad de Proyección Social de Psicología (UPS-PUCP). Texto preparado para la ceremonia de cierre de la experiencia colectiva realizada el 17 de agosto del 2001 en el campus de la universidad. 
Inés Kudó, Tesania Velásquez, Mónica Iza, Alicia Ángeles, César Pezo, Patricia Martínez

todos nosotros arrancar, llevar a cabo y concluir con orgullo y satisfacción íntima este proyecto colectivo.

Pensemos juntos ¿Qué cosa dentro de nosotros puede ser capaz de transformar una iniciativa de una díada, de un núcleo de miembros, en un empreño colectivo eficiente, imparable, sentido y pensado a cada momento, en un tiempo tan breve?

Compartamos y hagamos público nuestro origen.

- Sábado 23 de junio: terremoto y tsunami en el sur. Nos enteramos por los noticieros. El semestre académico está llegando a su fin, tiempo de exámenes, de correcciones. Todos están cansados.

- Viernes 6 de julio: segundo sismo, la tierra sigue temblando en el sur.

- Sábado 7 de julio: Inés Kudó y Tesania Velázquez, viendo las noticias, piensan que algo hay que hacer, que no nos podemos quedar pasivos. Son el emergente, la punta de lanza de un deseo que también tenían todos los que están acá.

- La preocupación por el otro y la urgencia de transformar esa preocupación en acción real toma cuerpo. Se echa a andar la convocatoria.

- $\quad$ Lunes 9 de julio: la Especialidad de Psicología hace suya esta iniciativa, tomamos el reto.

- Miércoles 11 de julio: la eficiencia se echa a andar, ya tenemos un proyecto organizado. La universidad lo hace enteramente suyo. Núcleos se reúnen para preparar coordenadas de intervención, ajustar costos, establecer contactos, convocar voluntades.

- Miércoles 18 de julio: todos los aquí presentes nos reunimos. Muchos ya se conocían, muchos otros no se conocían. Muchos trabajarán por primera vez juntos. Tenemos cosas claras, tenemos incertidumbres, ya vivíamos en nosotros mismos los 
temores y ansiedades que seguramente encontraríamos en el lugar de los hechos. Tomamos conciencia de que de repente no sabremos qué hacer allá. Nos apoyamos colectivamente.

- El sábado 21 de julio salen las primeras brigadas y el 14 de agosto regresan las últimas.

Todo es rápido. Una iniciativa de a dos se transforma en empeño colectivo e institucional. Cualquier impase encuentra solución. Cada quien pone sus recursos a disposición. Lo que alguien no puede hacer, otro lo hace. La dedicación y disponibilidad son libres. Todos aceptamos, sin recriminaciones ni demandas, lo que cada quien puede dar. La propuesta fue en libertad, la asunción del reto también.

Vuelvo a la pregunta inicial ¿Qué hay dentro de nosotros que somos capaces de un esfuerzo colectivo como éste?

No me voy a referir a la vocación de servicio, no al compromiso con una cierta idea de país. La preocupación por el otro define nuestro oficio.

Lo que ahora quiero subrayar es cómo la institución genera confianza. Es decir, dentro de nosotros, en nuestra mente, internalizada, el imaginario de un lugar confiable y esperanzador respecto a esta nuestra Especialidad de Psicología, hacia nuestra universidad.

Ése es el valor institucional que debemos rescatar y al que en esta oportunidad le hemos dado un giro cultural y político, porque la solidaridad es eso: una actitud que tiene que ver con la política y con la cultura. Es el espíritu que nos ha congregado a alumnos, docentes y egresados.

Otro elemento a subrayar es aquello que afirma Sartre a propósito de los grupos y que Serrat canta recogiendo los versos de 
Inés Kudó, Tesania Velásquez, Mónica Iza, Alicia Ángeles, César Pezo, Patricia Martínez

Machado: caminante, el camino se hace al andar. Los grupos se hacen y encuentran identidades en la praxis común. Los grupos nacen, crecen y se mueven dentro de instituciones. Pueden ser efímeros pero su obra engruesa la identidad de la institución.

Cada brigada y aquí todos juntos somos grupo. Nos hemos hecho grupo y este grupo mental pervivirá como parte de lo que somos y hacemos. Parte de lo que hemos hecho es revitalizar el espíritu institucional de solidaridad, preocupación por el otro y compromiso con las gentes necesitadas de nuestro país.

Todo lo que he dicho es obvio pero había que decirlo.

Termino agradeciéndoles a todos por esta renovación, todos deben aplaudirse a sí mismos, estar orgullosos y satisfechos de lo realizado. También tenemos que aplaudir a este sólido y eficiente equipo de cuatro mujeres formado por Mónica Iza, Alicia Ángeles, Tesania Velásquez e Inés Kudó. 